'llu. Revista de Ciencias de las Religiones

ISSN: $1135-4712$

http://dx.doi.org/10.5209/ILUR.61023

\title{
El temenos de Apolo y Aristeas en Metaponto. Una aproximación a la influencia de Delfos sobre la Magna Grecia ${ }^{1}$
}

\author{
David Hernández Castro²
}

Recibido: 13 de diciembre de 2017 / Aceptado: 13 de abril de 2018

Resumen. Durante la transición del siglo VI al siglo V a.C. la Magna Grecia experimentó grandes transformaciones sociales y políticas. El ascenso de la tiranía y los primeros avances democráticos produjeron una erosión del poder de la aristocracia y la consagración del Santuario de Delfos como la principal fuente de legitimidad de esta nueva centralidad política. El caso de Metaponto nos ofrece una oportunidad privilegiada para observar este proceso, ya que disponemos de varias fuentes, incluido un relato de Heródoto, que nos permiten acceder a la forma en la que el culto délfico cristalizó entre los metapontinos. La máxima expresión de este culto fue el temenos de Apolo y Aristeas, del que se ofrece una interpretación en este artículo, completada por la posible identificación de una base que habría formado parte del conjunto monumental y estaría representada en el registro numismático. Esta base se encuentra actualmente en las instalaciones del Parco Archeologico dell'area urbana di Metaponto.

Palabras clave: Delfos, ekklesiasterion, Heródoto, Magna Grecia, numismática.

\section{[en] The temenos of Apollo and Aristeas at Metapontion. An Approach to Delphi's Influence on Magna Graecia}

\begin{abstract}
During the period of transition from 6th century BC to 5th century BC, Magna Graecia experienced great social and political transformations. The rise of tyranny and the early democratic progress produced an erosion of the aristocracy's power and the consecration of the Sanctuary of Delphi as the foremost source of legitimacy for this new political centrality. The case of Metapontion provides a special opportunity to observe this process, because we have several sources, including a Herodotus story, that allow us to analyze the form of Delphic cult crystallized among the metapontians. The highest expression of this cult was the temenos of Apollo and Aristeas, that I provide an interpretation in this paper, supplemented by a possible identification of a base that had formed part of the monumental complex and would be represented in the numismatic record. This base is currently in the facilities of the Parco Archeologico dell'area urbana di Metaponto.
\end{abstract}

Keywords: Delphi, ekklesiasterion, Herodotus, Magna Graecia, numismatics.

1 Este artículo es resultado de una estancia de investigación de tres meses en la Escuela Española de Historia y Arqueología en Roma-CSIC, financiada por el Vicerrectorado de Investigación e Internacionalización de la UNED, y realizada durante el año 2017. Agradezco las sugerencias de los doctores Antonio Pizzo y José Ángel Zamora López, así como la colaboración y asistencia de todo el personal de la institución y del dottore Luigi Criscuolo y la dottoressa Pina Sangiorgio. Las tesis vertidas en este artículo son exclusiva responsabilidad del autor.

2 Investigador Predoctoral en Formación (FPI). Departamento de Filosofía. Facultad de Filosofía. Universidad Nacional de Educación a Distancia (UNED).

Correo electrónico: dhernandez@fsof.uned.es 
Sumario. 1. Introducción. 2. El temenos de Apolo y Aristeas. 3. La influencia de Delfos. 4. El temenos de Apolo y Aristeas en el contexto del conjunto monumental del ekklesiasterion. 5. Conclusiones. 6. Bibliografía. 7. Figuras.

Cómo citar: Hernández Castro, D. (2018), El temenos de Apolo y Aristeas en Metaponto. Una aproximación a la influencia de Delfos sobre la Magna Grecia, en 'Ilu. Revista de Ciencias de las Religiones 23, 111-128.

\section{Introducción}

Las excavaciones en el Parco Archeologico dell'area urbana di Metaponto realizadas en los años ochenta sacaron a la luz un recinto de forma trapezoidal que los arqueólogos no tardaron en identificar con el temenos de Apolo y Aristeas descrito por Heródoto en el libro IV de su Historia ${ }^{3}$. El temenos está localizado en el ángulo occidental del ágora, unos ochenta metros al suroeste de la imponente estructura del ekklesiasterion ${ }^{4}$. Aunque los restos recuperados se corresponden con bastante fidelidad a la descripción de Heródoto, algunos aspectos son todavía objeto de discusión, debido fundamentalmente a ciertas dificultades de interpretación del texto. Una de ellas está relacionada con la existencia o no de una estatua de Apolo dentro del recinto sagrado, que Mertens-Horn ha creído posible identificar con el fragmento de un torso aparecido cerca de las ruinas del Tempo de Apolo Licio, actualmente expuesto en el Museo di Potenza ${ }^{5}$. Aunque el texto de Heródoto no es concluyente, se ha encontrado un fuerte argumento a favor de la existencia de esta estatua en la imagen de Apolo representada en un grupo de acuñaciones que forman parte de las primeras series en doble relieve de Metaponto ${ }^{6}$. La imagen de estas monedas concuerda con algunos detalles que Mertens-Horn ha reconocido en el torso de Potenza ${ }^{7}$. Estas representaciones nos muestran una figura de Apolo sosteniendo un arco en su mano izquierda y una rama de laurel en su mano derecha ${ }^{8}$. En una de las acuñaciones, el extremo de la rama se encuentra enclavado en lo que hasta ahora ha sido identificado como un altar (fig. 1), quizás, el altar de Apolo descrito por Heródoto, de cuya existencia las excavaciones han despejado cualquier $\mathrm{duda}^{9}$. En este artículo propondré una identificación alternativa para esta base en la que estaría enclavada la rama de laurel. Se trata de una pieza que se encuentra actualmente en las instalaciones del Parco Archeologico dell'area urbana di Metaponto (figs. 2 y 3), y que coincide con la imagen representada en la moneda al menos en cuatro aspectos fundamentales: la

$3 \quad$ Hdt., IV.15; De Siena 1992, 116, 1998, 156-159, 167-168; Mertens 1998, 130; De Juliis 2001, 170-173; Cerchiai 2002, 141; Giacometti 2005, 43; y Lane 2009, 205-209.

4 Para una visión detallada de la zona del ágora, el temenos y el ekklesiasterion, cfr. Adamesteanu 1979a, 1979b; De Siena 1979; Mertens 1979, 1982; De Siena 1992, 116-117; Mertens y Greco 1996, 252-254; De Siena 1998, 150-159; Mertens 1998; Giardino y De Siena 1999, 350-359; De Juliis 2001, 158-173; De Siena 2001, 29-35; Mertens 2001, 63-68; Cerchiai 2002, 140-141; Greco 2006; Mertens 2006, 161-163, 332-337.

5 Mertens-Horn 2001, 74-75.

$6 \quad$ Head 1911, 76; Lehmann 1946, 8, 33; Giannelli 1963, 62-65; Stazio 1974, 83-84, 1999, 458-459; Rutter 2001, 132; De Juliis 2001, 61; y Giacometti 2005, 45.

Mertens-Horn 2001, 75.

Noe 1931, Plate XXIV, $\mathrm{n}^{\circ}$ 314-320.

$9 \quad$ La base fue identificada como un «altar» por Poole $(1873,242)$, Head $(1911,76)$ y Noe $(1931,8)$. La identificación de este altar con el altar descrito por Heródoto y con el que apareció en las excavaciones de Metaponto, en De Siena 1998, 159; De Juliis 2001, 61; y De Siena 2001, 35. 


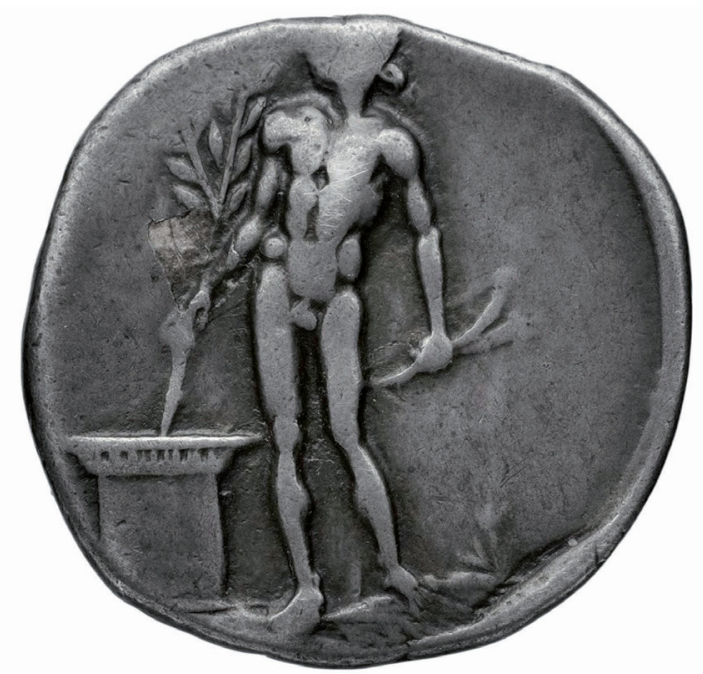

Figura 1. Noe 1931, Plate XXIV, no 319. Bibliothèque nationale de France, département Monnaies, médailles et antiques, Fonds général, no 1143 (imagen: BNF domaine public).

forma, el tamaño, el relieve y la disposición del agujero de su cara superior. La confirmación de esta identificación constituiría un argumento concluyente a favor de la existencia de la estatua de Apolo. Tanto la base, como la estatua, podrían haber sido desplazadas del temenos en una remodelación posterior y reubicadas en el entorno del Templo de Apolo Licio. El desarrollo del análisis conducirá, por otro lado, a una interpretación más amplia del temenos de Apolo y Aristeas, cuyo origen hay que buscar en el impacto de Delfos sobre la Magna Grecia, y cuya evolución debe contemplarse a la luz de su vínculo estructural (al menos, en sus primeras etapas) con el ekklesiasterion.

\section{El temenos de Apolo y Aristeas}

Los primeros restos monumentales del temenos se remontan a las décadas iniciales del siglo $\mathrm{V}$ a.C., y son por tanto contemporáneos a la primera remodelación del ekklesiasterion que ocupa la esquina norte del ágora (fig. 4). El temenos experimentó diversas transformaciones hasta el siglo I a.C., lo que constituye un caso raro de pervivencia monumental y cultual de la Metaponto griega, en un contexto donde incluso el templo de Apolo Licio estaba ya en ruinas. Los restos que podemos datar de la primera fase son, básicamente, un altar, un pozo circular, y once metros al sudoeste, una gran base de bloques de piedra, con toda probabilidad, destinada a sostener una estatua de grandes proporciones. En las proximidades del pozo y el altar se han encontrado una cantidad considerable de hojas de laurel de bronce. Más adelante, a finales del siglo III a.C., el altar fue rodeado por un recinto de piedra rectangular, sin techo y abierto por el lado este, y esta es la fecha en la que probablemente se construyó también un segundo recinto de forma trapezoidal que tenía como finalidad delimitar toda el área sagrada. La última transformación se produjo entre los siglos II 


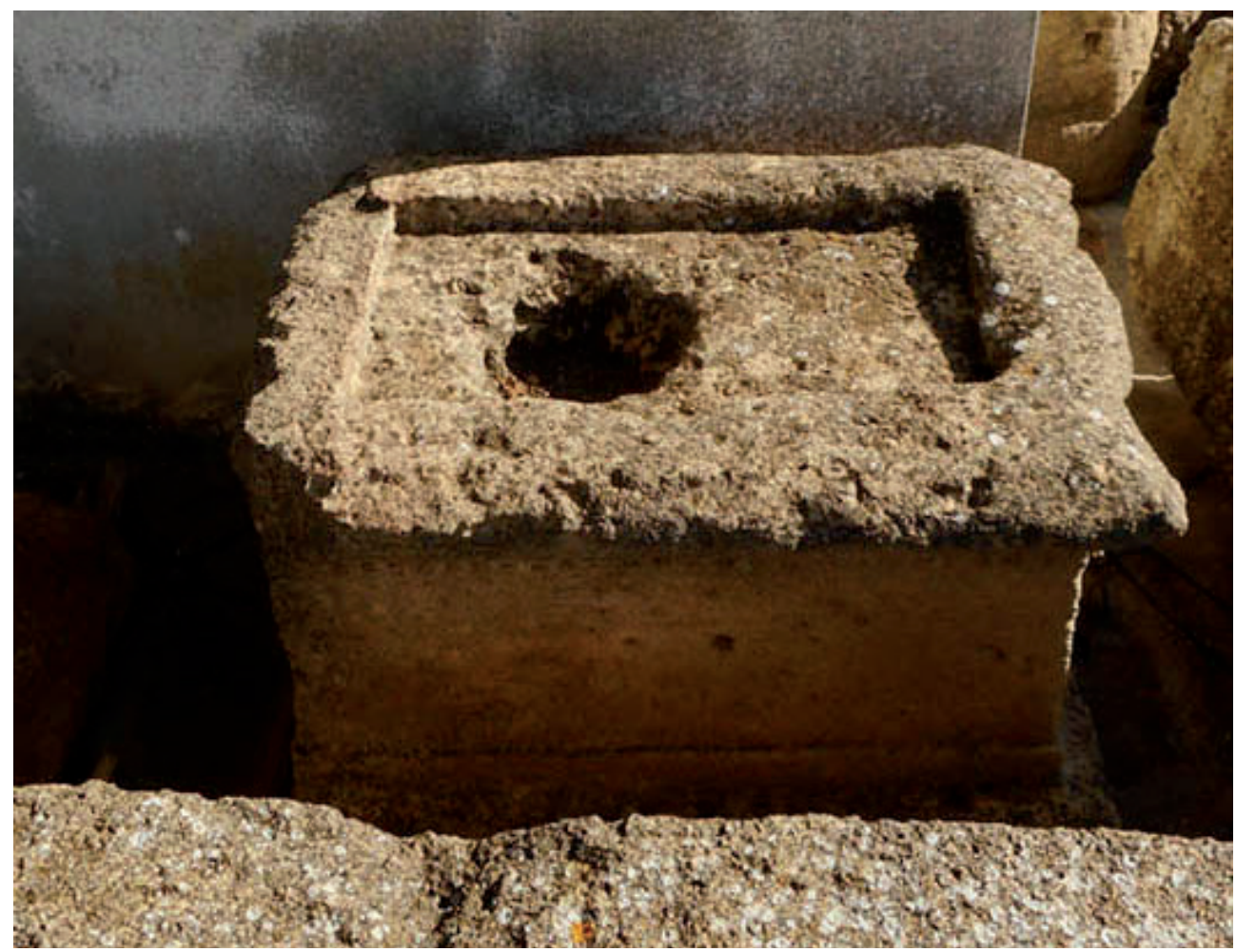

Figura 2: Base. Parco Archeologico dell'area urbana di Metaponto (fotografía: David Hernández Castro).

y I a.C., cuando fueron añadidas un grupo de estancias cuya disposición parece concordar con la de un hestiatorion ${ }^{10}$.

El relato de Heródoto nos ha permitido llegar en la interpretación del temenos mucho más lejos de lo que nos hubieran permitido los restos de las excavaciones. Además, contamos también con una breve anécdota de Ateneo (pero que se remonta a Teopompo, segunda mitad del siglo IV a.C.) cuya trama transcurre en este escenario, y con la acuñación monetaria que he mencionado al principio de este artículo, datada a mediados del siglo V a.C. ${ }^{11}$

Heródoto describe que Aristeas de Proconeso, un personaje cuya biografía reviste tintes legendarios ${ }^{12}$, se apareció a los metapontinos para ordenarles que levantaran

10 De Siena 1998, 156-159; De Juliis 2001, 170-173.

11 Ath., XIII.83 605C-D (=Theopomp.Hist., fr. 248, Jacoby, FGH 115). La datación de Noe, en torno al 480 a.C., fue rebajada posteriormente al 450-440 a.C. (Stazio 1974, 78) o 440-430 (Stazio 1999, 458).

12 El estudio más completo sobre Aristeas de Proconeso sigue siendo Bolton 1962. Otras aproximaciones en Dodds 1951, Colli 1977 y Bremmer 1983. Sabemos muy poco acerca de su existencia histórica. No obstante, parece que fue un personaje real, cuya actividad se desarrolló en torno al siglo VII a.C., y que escribió un poema llamado Arimaspeia donde se relataban sus viajes por la tierra de los hiperbóreos. La tradición lo relaciona estrechamente con Apolo, y ya en los tiempos de Heródoto, se contaban historias sobre su capacidad para la bilocación o para adoptar la forma de un cuervo. Bremmer no acepta la hipótesis chamánica de Dodds. En su opinión, no hay que confundir los relatos acerca de la capacidad para viajar del alma libre con una supuesta influencia del chamanismo. Cf. Dodds 1951, 135-178 y Bremmer 1983, 25-53. 


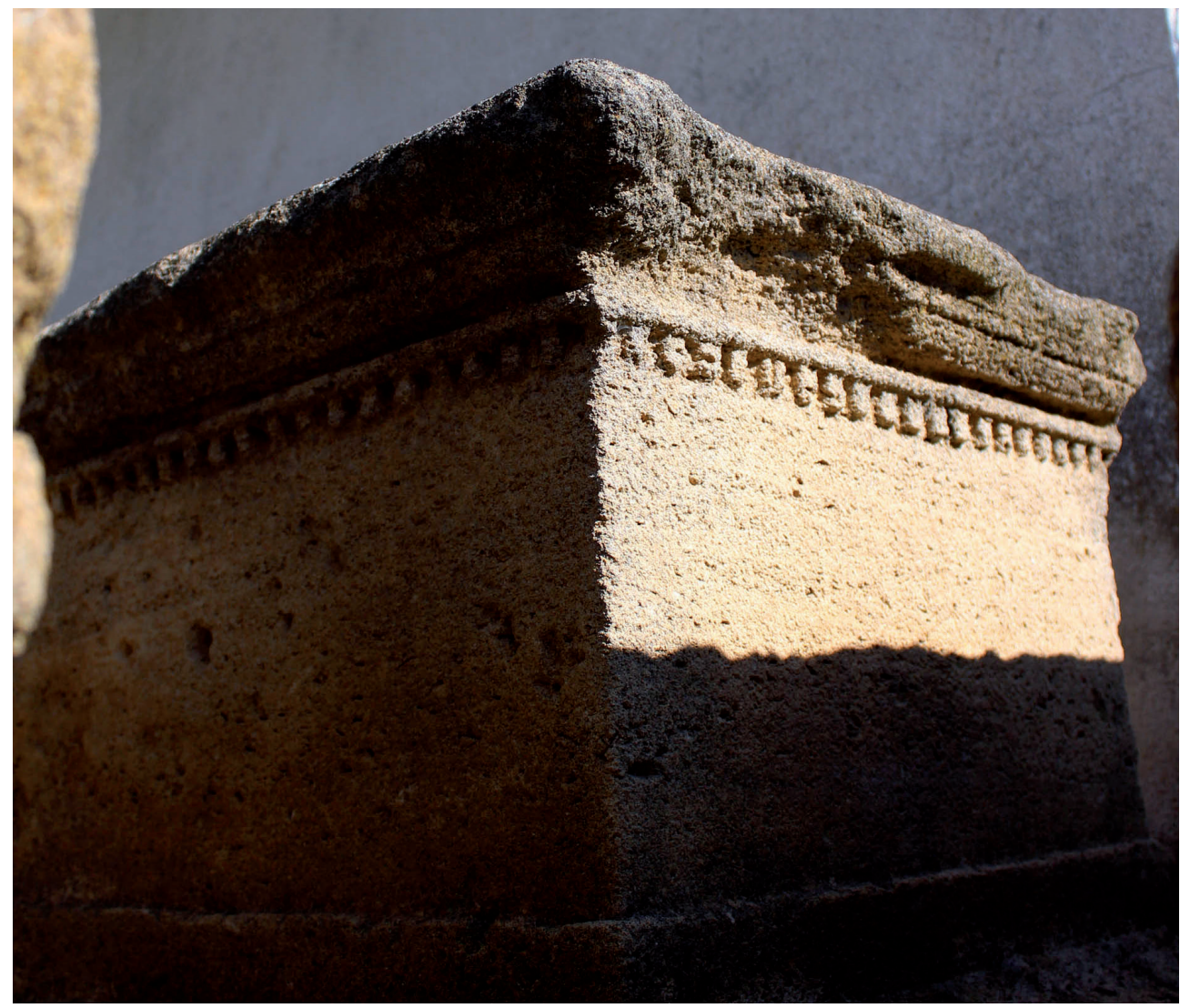

Figura 3. Base. Parco Archeologico dell'area urbana di Metaponto (fotografía: Luigi Criscoulo).

un altar en honor de Apolo, y al lado de dicho altar, una estatua que debería llevar su propio nombre. La razón de todo esto, habría dicho Aristeas, era que los metapontinos debían conmemorar de esta manera el hecho de ser los únicos italiotas a cuyo país había llegado hasta la fecha Apolo. Los metapontinos, tras la debida consulta a Delfos, decidieron cumplir el mandato de Aristeas y levantar en el ágora la estatua en su nombre y el altar dedicado al dios. El relato de Heródoto habla también de una

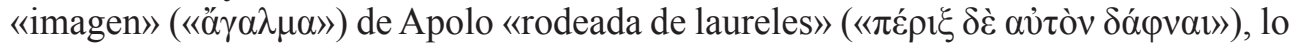
cual coincide con las hojas de laurel de bronce que se han encontrado en las excavaciones $^{13}$. No ha habido grandes problemas a la hora de identificar el lugar donde debía levantarse la estatua de Aristeas con la gran base de bloques de piedra que ha sido localizada al sudoeste del altar. Pero a partir de aquí, la narración de Heródoto se vuelve algo imprecisa, y no está claro si lo que describe junto al altar es un árbol de laurel de bronce, o un árbol de laurel de verdad, decorado con hojas de bronce, o una segunda escultura, esta vez, del propio Apolo, que es lo que parece desprenderse de la acuñación metapontina y de la palabra «ő $\gamma \alpha \lambda \mu \alpha »$. O tal vez, las tres cosas al mis-

13 De Siena 1998, 157. 
mo tiempo. Es decir, un árbol de verdad, una rama de laurel, decorada con hojas de bronce, y una estatua de Apolo. Por empezar desde el principio, abordaré el problema relacionado con las dudas acerca de la existencia de esta segunda estatua.

Hay en la descripción de Heródoto, cuando se refiere a lo que los metapontinos construyeron en honor de Apolo, un cambio de registro que ha desconcertado a los intérpretes. En la primera parte del relato, utiliza la palabra « $\beta \omega \mu$ ó $»$ : «ordenándoles

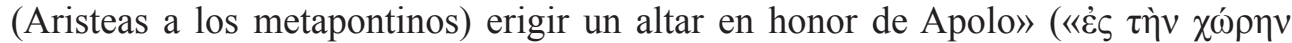
$\kappa \varepsilon \lambda \varepsilon \tilde{v} \sigma \alpha \imath \beta \omega \mu$ òv 'A $\left.\boldsymbol{c o ́}^{\prime} \lambda \lambda \omega v o \varsigma »\right)$. Hasta aquí, el asunto no ofrece dudas. Lo que sigue a continuación es la consulta de los metapontinos a la Pitia, cuyo consejo de obedecer el mandato de Aristeas decidieron poner en práctica ( $(\pi 0 \imath \tilde{\eta} \sigma \alpha \iota \dot{\varepsilon} \pi \imath \tau \varepsilon \lambda \varepsilon \dot{\varepsilon} \alpha »)$. Y es entonces, en la última parte de su relato, cuando nuestro autor cambia de registro. En lugar de « $\beta \omega \mu$ ó $»$, es decir, un altar, utiliza la palabra «ő $\gamma \alpha \lambda \mu \alpha »$, cuya traducción más adecuada es la «imagen» de un dios, es decir, una estatua: «Y hoy en día una

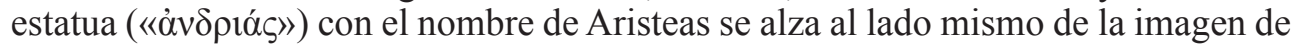

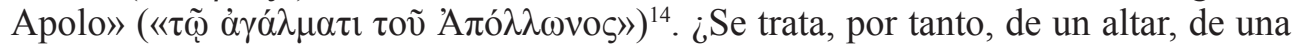
estatua, o de ambas cosas al mismo tiempo?

En cuanto al árbol de laurel, el testimonio de Ateneo nos ofrece algunos detalles complementarios. La anécdota está relacionada con una bailarina llamada Farsalia, cuya desgracia se desencadenó el día en que cometió la imprudencia de pasearse por el ágora de Metaponto después de aceptar una corona de laurel de oro. En cuanto la

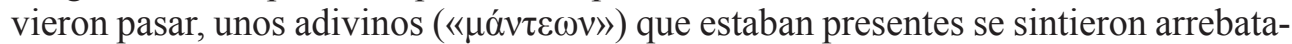

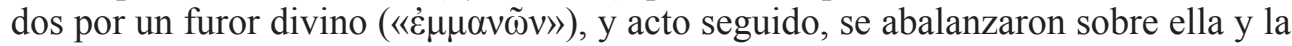
despedazaron. Los hechos fueron investigados, pero quedó claro que la causa de la muerte había sido la corona del dios, porque justo antes de que estallara el furor de

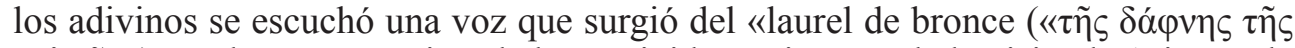
$\chi \alpha \lambda \kappa \tilde{\eta} \varsigma »)$ que los metapontinos habían erigido en tiempos de la visita de Aristeas de Proconeso» ${ }^{15}$.

La mención de Ateneo a los adivinos y el laurel de bronce, junto a los restos hallados en las excavaciones y lo que sabemos de las prácticas que se ejercían en los santuarios de Apolo, ha reforzado la tesis de que nos encontramos ante un manteion, un lugar donde no solo se celebraban sacrificios rituales en honor a Apolo, sino también otras actividades relacionadas con la adivinación oracular ${ }^{16}$. En cuanto al «laurel de bronce», las excavaciones han hecho aflorar frente al altar un agujero donde algunos autores han creído reconocer el lugar donde estaba fijado este árbol de laurel ${ }^{17}$.

Por otro lado, la identificación con un «altar» de la base sobre la que descansa el extremo de la rama que Apolo sujeta en la acuñación metapontina ha sido ampliamente aceptada. Sin embargo, en las instalaciones del Parco Archeologico dell'area urbana di Metaponto se conserva una base de piedra que coincide con la que aparece representada en la acuñación en cuatro aspectos fundamentales: el tamaño, la forma, el relieve decorativo, y la disposición del agujero labrado en la piedra donde podría

Hdt., IV.15.

Ath., XIII.83 605C-D. Plutarco nos da otra versión igualmente cruel de la historia (pero más indulgente con el papel de los adivinos), según la cual el asesinato se habría producido cuando Farsalia bailaba en el templo de Apolo, mientras que los culpables habrían sido unos jóvenes que trataban de apoderarse de la corona de oro. Cfr. Plu., Moralia, 397F-398A.

16 De Siena 1998, 158; De Juliis 2001, 170.

17 De Siena 1998, 158; De Juliis 2001, 61, 171; Giacometti 2005, 43. 
haber estado fijada la rama de laurel (figs. 2 y 3). La coincidencia en el tamaño y la forma es bastante notable, dado que el contorno de ambas piezas reproduce una misma apertura tanto en el borde superior como en la base. Pero más notable aún es la semejanza del relieve decorativo, geométrico en la parte superior, y liso, en la parte inferior. Finalmente, incluso la disposición del agujero de la cara superior nos ofrece una información relevante para esta identificación. No está en el centro, sino ligeramente desviado hacia uno de los extremos, lo cual coincide con el dibujo de la moneda, donde el punto donde descansa la rama se encuentra algo más cerca de la rodilla de Apolo que del otro extremo de la pieza. Pero esta desviación del agujero tiene otra implicación importante. Si se tratara de la base de una estatua, este agujero, que estaría destinado a sujetar la fijación de la planta, debería estar en el centro, y no desviado hacia uno de los extremos.

La suma de todos estos aspectos contribuye a respaldar la tesis de que esta pieza hallada en el área arqueológica de Metaponto sea efectivamente la base representada en la acuñación que los metapontinos realizaron a mediados del siglo $\mathrm{V}$ a.C. En este

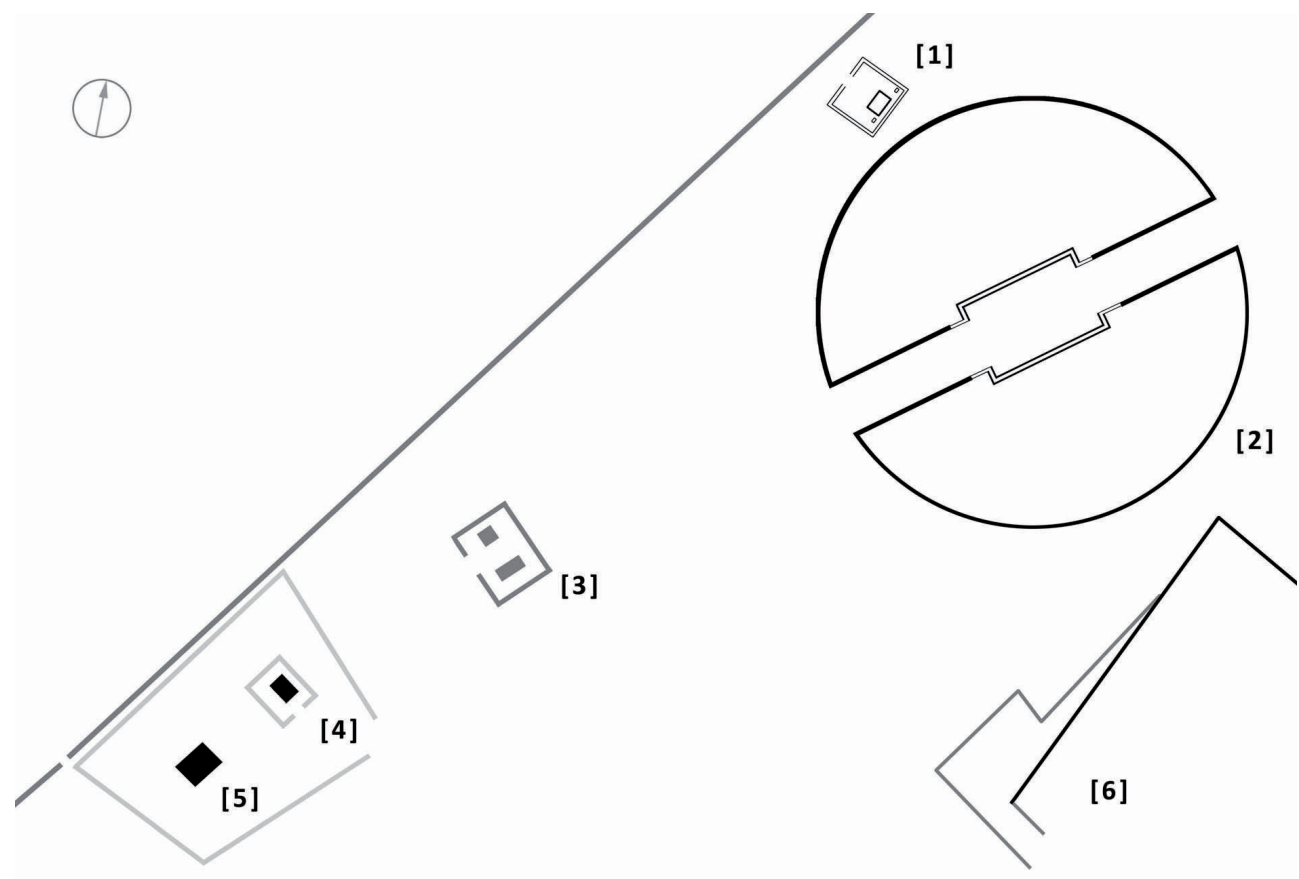

[1] Altar de Zeus Agoraios

[2] Ekklesiasterion

[3] Temenos anónimo

Fuente: Elaboración propia a partir de De Siena (2001) y Mertens $(2001,2006)$
Ágora de Metaponto Siglo V a.C.

Siglo IV a.C.

Siglo III a.C.

Figura 4. Ágora de Metaponto. Elaboración propia a partir de De Siena 2001 y Mertens 2001, 2006. 
caso, no habría razones para poner en duda que la estatua de Apolo y la rama de laurel fueron tan reales como ella. Por otro lado, dado que esta base parece mantener cierta proporción con la base representada en la moneda, cabe esperar que ocurriera lo mismo con la rama de laurel. Sin embargo, el tamaño de la rama no termina de adecuarse a la expresión de Heródoto de que la imagen de Apolo estaba «rodeada» de laureles, lo que se avendría mejor con la idea de que además de esta rama la zona contaba con un verdadero árbol de laurel.

Conviene ahora que prestemos algo de atención a la descripción que Noe realizó del grupo de acuñaciones al que pertenece la moneda que estamos analizando, porque esta descripción nos plantea varios problemas que debemos abordar. Se trata de la tabla Plata XXIV, que recoge las primeras acuñaciones en doble relieve de Metaponto. Por el anverso, estas series son bastante similares. Incluyen siempre una espiga de cebada, y algunas de ellas, en los lados de la espiga, un saltamontes y la inscripción «META». Las diferencias más importantes se encuentran en el reverso. Aquí, dos de las series muestran una representación del dios fluvial Aqueloo, otras dos, de Heracles, y las últimas siete, del dios Apolo ${ }^{18}$. Noe propuso datar con este orden las emisiones a partir de ciertas características técnicas de las acuñaciones ${ }^{19}$. Entre las siete series que representan al dios Apolo, la diferencia más importante es que solo en una de ellas, la $\mathrm{n}^{\circ} 319$, la rama de laurel aparece fijada sobre una base. En casi todas las demás, el extremo de la rama se extiende hasta el suelo (fig. 5) ${ }^{20}$. El problema es que en una de las dos series de Heracles, la n 312 (fig. 6), nos encontramos con una base muy similar a la que aparece representada en la serie de Apolo. No hay, en este caso, ninguna rama de laurel. En su lugar, Heracles sostiene en su mano derecha una pátera con la que parece estar vertiendo una libación sobre la base. Esto llevó a Noe a extraer dos conclusiones. La primera, que esta base era un altar. Y la segunda, que el altar representado en la serie $\mathrm{n}^{\mathrm{o}} 319$, y que no vuelve a aparecer en ninguna de las otras series de Apolo, era una «obvious reminiscence» del altar representado en la anterior acuñación de Heracles ${ }^{21}$. Debemos tener en cuenta, además, que en las dos series que representan al dios Aqueloo también esta divinidad aparece sosteniendo una pátera, aunque en este caso, sin rastro de nada que pueda identificarse con un altar (fig. 7). Contienen, sin embargo, una inscripción bastante significativa: «AKE $\Lambda$ OIO AE $\Theta \Lambda \mathrm{ON} »$, lo que puede aludir a Aqueloo en su faceta de combatiente, o al premio de un combate, o mucho más probable, como ocurría en Acarnania, a unos juegos celebrados en honor del $\operatorname{dios}^{22}$.

18 Aqueloo, $n^{\circ} 311$ y n 311 1/2; Hércules, no 312 y 313; Apolo, no 314-320. El reverso de la no 310 presenta cinco granos de cebada formando una estrella.

19 Noe 1931, 4-8.

20 La excepción es la $n^{\circ} 320$, en la que ha desaparecido la rama de laurel. En su lugar, Apolo tiene la mano derecha sobre la cadera. Se trata, según Noe, de una moneda bastante rara, un hemi-estatero (la división de un estatero por la mitad, en lugar de en tres partes, como era habitual), que pudo haberse acuñado como consecuencia de una corta influencia de Tarento sobre la ciudad. Cfr. Noe 1931, 8, 11.

$21 \quad$ Noe 1931, 8 .

22 Head 1911, 76; Noe 1931, 6; Giannelli 1963, 77-79; Lacroix 1965, 82; Stazio 1974, 80-81, 1999, $458-459$. Como premio destinado al vencedor en los juegos, cfr. Franke-Hirmer 1966, 65. Se ha planteado también como alternativa la conmemoración de una obra de saneamiento. Esta interpretación estaría apoyada en un pasaje de Pausanias (I.41.2) donde se menciona que Teágenes, tirano de Mégara, erigió por esta razón un altar en honor del dios Aqueloo, y también en unas grandes obras de saneamiento que han aflorado en Metaponto gracias al trabajo de las excavaciones. Cfr. Uggeri 1969, 51-71. Estas obras, sin embargo, son de época tardo-arcaica, y no se ajustan a la datación de las acuñaciones. Para el debate, cfr. Stazio 1974, 80-81, 1999, 458-459 y De Juliis 2001, 74-75. 


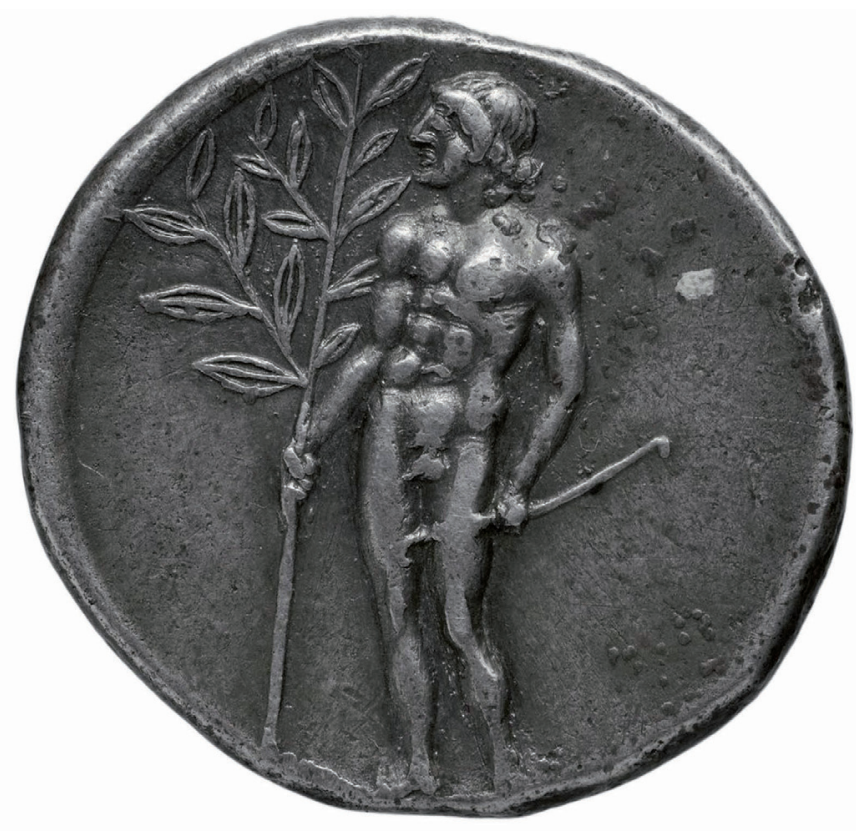

Figura 5. Noe 1931, Plate XXIV, no 315. Bibliothèque nationale de France, département Monnaies, médailles et antiques, Fonds général, nº 1144 (imagen: BNF domaine public).

La interpretación de Noe es muy sugerente, pero en lo que se refiere a la base representada en la serie $n^{\circ} 319$ implicaría que en lugar de una base nos encontramos ante un altar, y no precisamente ante el altar de Apolo, sino ante una «reminiscence» del altar de Heracles. Creo, sin embargo, que es posible reorientar sus argumentos en una dirección compatible con la interpretación de todos los autores que han propuesto relacionar esta moneda con el temenos de Apolo y Aristeas. Pero para abordar este asunto, es conveniente empezar a ordenar todo el material que hemos puesto hasta ahora sobre la mesa.

\section{La influencia de Delfos}

Según mi interpretación, el diseño original del temenos constaba de un altar, un árbol sagrado de laurel (situado en frente), una estatua de Apolo, con la disposición que aparece representada en las acuñaciones, y una estatua de Aristeas, que estaría ubicada once metros al sudoeste. Las hojas de laurel de bronce que se han encontrado en las excavaciones probablemente fueran un ornamento ritual de la rama, tal vez del árbol, o de las dos cosas al mismo tiempo. La «imagen» de Apolo estaría en algún lugar junto al altar y el árbol sagrado, orientada hacia el este, pero con la cabeza girada, como aparece representada en las acuñaciones, mirando hacia su derecha, en la dirección de la estatua de Aristeas de Proconeso. De Juliis señala que en la sistematización más reciente del temenos parece difícil encontrar junto al altar un lugar apropiado para la estatua de Apolo, lo que le lleva a concluir que, o bien nunca estu- 


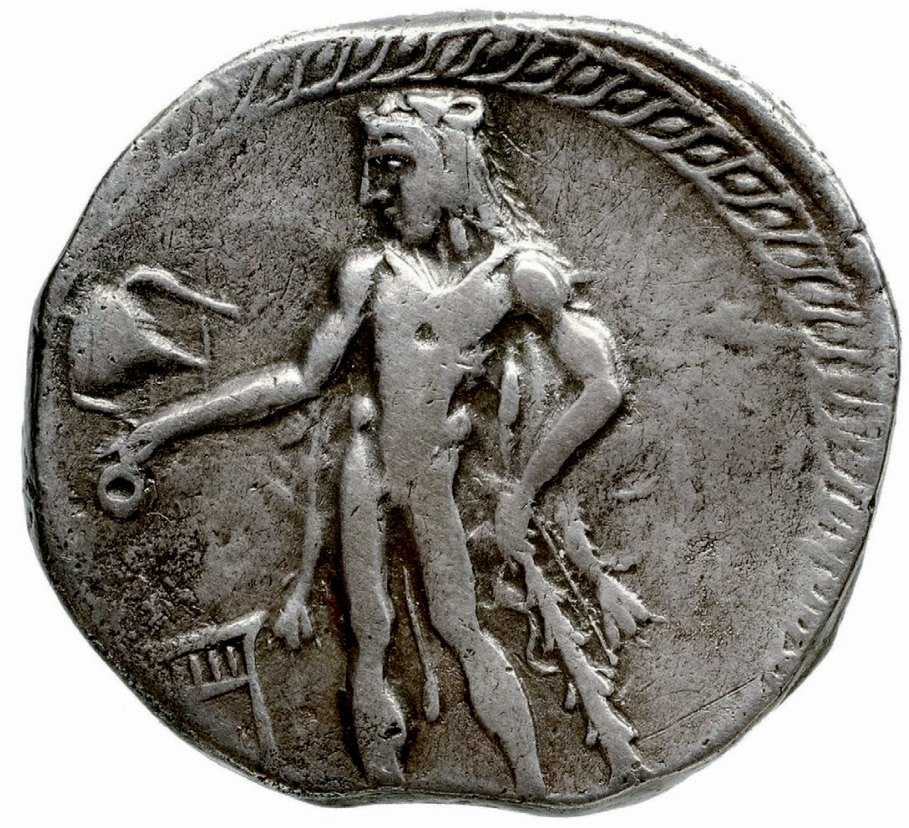

Figura 6. Noe 1931, Plate XXIV, nº 312. Bibliothèque nationale de France, département Monnaies, Luynes, $n^{\circ} 467$ (imagen: BNF domaine public).

vo en aquella parte, o bien fue trasladada en el curso de una reestructuración posterior $^{23}$. Esto coincidiría con el hallazgo del torso que Mertens-Horn atribuye a Apolo en las inmediaciones del Templo de Apolo Licio. No es incompatible la existencia del árbol de laurel, que estaría enraizado en el agujero que todavía es visible frente al altar, y la rama de laurel que la estatua de Apolo sostenía en la mano. De hecho, es posible que esta rama fuera elegida de entre las ramas del árbol sagrado, que se trataría, en este caso, de un árbol de verdad. La rama se renovaría coincidiendo con la festividad del dios, y sería embellecida con las hojas de laurel de bronce. Otras series de monedas acuñadas en Metaponto apuntan a la existencia de un culto a Apolo Karneios (Kópveios), cuya festividad se celebraba durante el verano ${ }^{24}$. Sin embargo, para Giacometti y De Juliis, la presencia del laurel sugiere que Apolo fue adorado en este santuario bajo el epíteto de Daphnephoros $(\Delta \alpha \varphi v \eta \varphi o ́ p o \varsigma)^{25}$. En este caso, es oportuno recordar la festividad délfica del Septerion, en la que una procesión de jóvenes se dirigía al Valle del Tempe para cortar de un árbol de laurel sagrado unas ramas que luego portaban de regreso a Delfos ${ }^{26}$. Esta festividad se celebraba una vez cada ocho años, y las ramas de laurel se utilizaban para realizar las coronas que se entregaban de premio durante los Juegos Píticos. Quizás, en el caso de Metaponto, las ramas del árbol de laurel se utilizaran con un fin parecido, lo cual parece corresponderse con la hipótesis de Mertens de que el cercano ekklesiasterion se utilizara en

\footnotetext{
De Juliis 2001, 172.

Noe 1931, Plate XXVI, no 334-344; Lane 2009, 210-211.

De Juliis 2001, 61; Giacometti 2005, 43.

Fontenrose 1980, 453-461; Plu. Moralia, 296C, 421C y 1136B.
} 


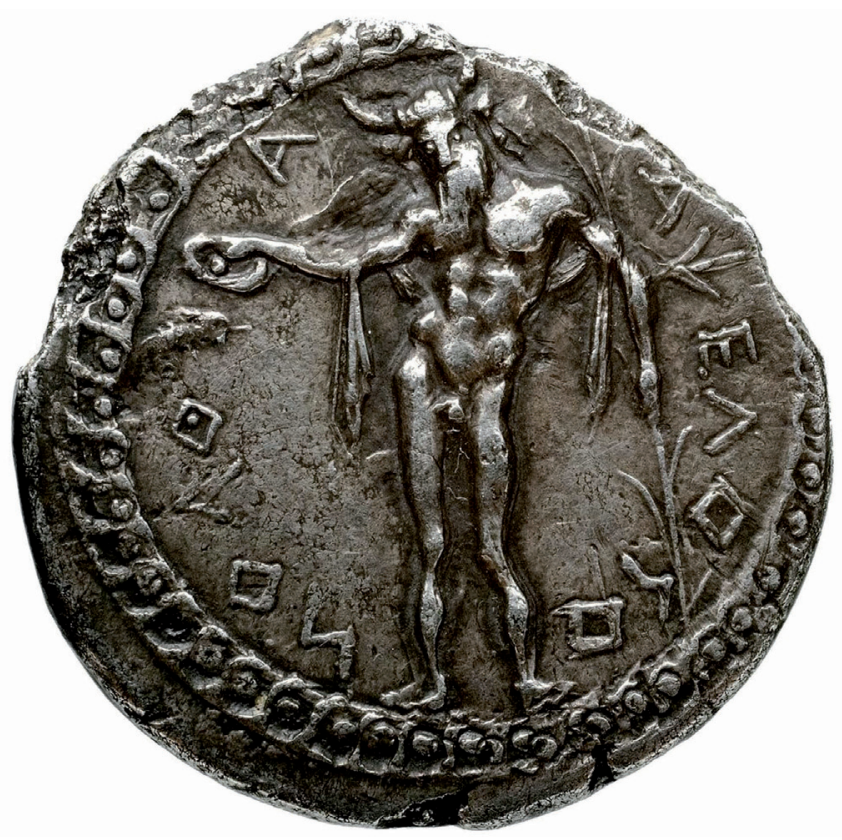

Figura 7. Noe 1931, Plate XXIV, nº 311. Bibliothèque nationale de France, département Monnaies, Luynes, $n^{\circ} 466$ (imagen: BNF domaine public).

algunos momentos para celebrar competiciones musicales y atléticas ${ }^{27}$. El altar de Apolo se encuentra a unos ochenta metros al suroeste del ekklesiasterion, y llama poderosamente la atención el hecho de que el corredor central que atraviesa esta estructura (en el que, según Mertens, se celebraban los certámenes gimnásticos), estuviera orientado directamente hacia el altar ${ }^{28}$. Es decir, cuando uno se ubicaba en el centro de la orchestra del ekklesiasterion, la única vista posible del ágora, estrechada por el corredor de su eje principal, era la que apuntaba directamente hacia el conjunto formado por el altar de Apolo, la imagen del dios, el árbol de laurel, y la estatua de Aristeas que se levantaba sobre la gran base del fondo ${ }^{29}$. En relación con todo esto, disponemos de un fragmento de Simónides donde encontramos una descripción

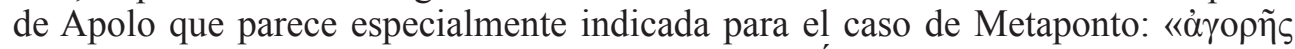
$\kappa \alpha \lambda \lambda \imath \chi o ́ \rho o v ~ \pi \rho v ́ \tau \alpha v \imath v »$, es decir, Apolo, «prítano del Ágora, lugar de nobles coros de danzas $\gg{ }^{30}$. La propuesta de interpretación de Mertens apunta precisamente a la posi-

27 Mertens 2006, 162, 337.

28 Mertens llamó también la atención sobre este aspecto. Cfr. Mertens 1998, 130.

29 Entre el ekklesiasterion y el temenos de Apolo hay un recinto rectangular con una doble base que inicialmente fue identificado por Adamesteanu como el temenos descrito por Heródoto (1979a, 310ss). La ampliación posterior de las excavaciones corrigió este punto de vista, $c f r$. De Siena 1998, 159, n. 45. En relación a este nuevo recinto, Mertens habló de «datazione incerta» $(1998,130)$. Posteriormente, lo encuadró a finales del siglo IV a.C. Cfr. su propuesta reconstructiva del santuario y el ágora en Mertens 2001, 51, fig. 50, 52, fig. 52, y 63, fig. 69; 2006, 157, fig. 271. La fig. 275 de 2006, 159 lo sitúa en el siglo V a.C., pero debe tratarse de un error, ya que incluye, entre otros aspectos, el recinto del altar del temenos de Apolo, datado en el siglo III a.C.

30 Simon., Epigrammata, 164.2 (=VI.212). Marcel Detienne quiso subrayar las connotaciones políticas del fragmento: «"le Prytane de l’Agora”, celui qui commande en maître et semble régner sur l’Assemblée» $(1998,125)$. No obstante, señaló que era necesario atender a las relaciones complejas que Apolo tiene con los diferentes 
bilidad de que la estructura del ekklesiasterion fuera diseñada en su origen para representar una danza coral: el plato fuerte serían dos coros, que entrarían a la orchestra por cada uno de los extremos del corredor principal (dromos), y que en el momento del agon se moverían uno enfrente del otro ${ }^{31}$.

La importancia de los juegos y las celebraciones gimnásticas en el Metaponto de la segunda mitad del siglo V a.C. está atestiguada por otras dos fuentes: la primera tiene una importancia excepcional, porque en ella se anudan la celebración gimnástica, la interpretación coral y la impronta del Santuario de Delfos. Se trata de un epinicio de Baquílides dedicado a la victoria del joven Alexidamo de Metaponto en la prueba de lucha de los Juegos Píticos ${ }^{32}$. La datación de esta oda es bastante incierta, pero en todo caso se compuso en las décadas anteriores a la mitad del siglo $\mathrm{V}$ a.C. Baquílides rinde tributo a la diosa Victoria, y celebra que gracias a ella Metaponto

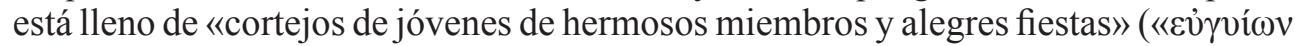

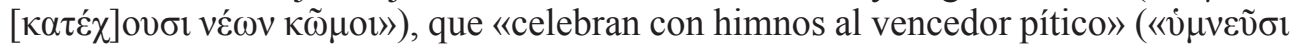

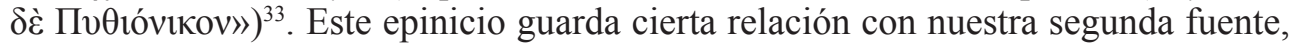
que es ya contemporánea a la visita de Heródoto y al grupo de acuñaciones de Apolo. Se trata de la cabeza de la escultura de un luchador, que debió formar parte de una composición de grandes proporciones en la que se representaba el combate entre dos atletas. Mertens-Horn ha realizado una propuesta de reconstrucción de esta figura y la ha relacionado con los actos de conmemoración de la importante victoria de Alexidamo $^{34}$.

Es difícil sostener que los metapontinos eligieran el ágora como el lugar más apropiado para instituir el temenos de Apolo y Aristeas de forma accidental. Por el contrario, debieron tener en cuenta el contexto significativo que lo rodeaba, y los elementos más importantes de este contexto eran el ekklesiasterion y la propia dimensión pública que implicaba el ágora. Todo lo que sabemos de él apunta claramente en la dirección de Delfos, algo en lo que no parece necesario que debamos insistir, una vez que disponemos de un testimonio tan valioso como el de Heródoto. El culto a Apolo siempre fue muy importante en Metaponto $^{35}$, pero creo que la aparición de este temenos a inicios del siglo $\mathrm{V}$ a.C. tiene que ser puesta en relación con las transformaciones políticas que en esta época se estaban produciendo en Delfos y con el incremento de su influencia sobre la Magna Grecia ${ }^{36}$. Michael Scott habla de un «overwhelming deluge of monuments and oracular consultations», y señala que esta popularidad creciente «none more so tan among the Greeks of the western Mediterranean world of Magna Graecia» ${ }^{37}$. No cabe duda de que los Juegos Píticos se convirtieron en una de las expresiones más importantes de este proceso, y es muy posible que uno de los propósitos del temenos de Apolo y Aristeas (aunque no el único) fuera poner bajo la advocación del dios de Delfos las competiciones gimnásticas que tradicionalmente se

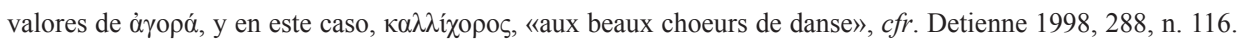
Para la dimensión política de Apolo Daphnephoros, cfr. Detienne 1998, 126-127, 290, n. 139; y Knoepfler 1988, 385-389.

31 Mertens 2006, 162, 337. Cfr. Mertens 1982, 102ss.; De Siena 2001, 32; Mertens 2001, 65; y Greco 2006, 330331.

$32 \quad$ B. Ep., 11.

33 B. Ep., $11.10-15$.

34 Mertens-Horn 2001, 79-80.

35 Basta recordar el Santuario de Apolo Licio, aunque no solo. Cfr. Lane 2009, 198-212.

36 Scott 2014, 121-124.

37 Scott 2014, 122. La influencia de Delfos sobre Metaponto ya fue señalada por De Siena 1998, 167-168. 
celebraban en la pista central del ekklesiasterion. Tal vez estos juegos nacieron vinculados al dios Aqueloo, y no sería extraño que los metapontinos acudieran también al patrocinio de Heracles ${ }^{38}$. Pero la importancia que el temenos de Apolo y Aristeas alcanzó ya en los tiempos de la visita de Heródoto es un testimonio de la posición central que el dios de Delfos tuvo que terminar ocupando en esta y otras dimensiones de la vida pública de los metapontinos, como parece confirmar el peso cada vez mayor que fue adquiriendo en las nuevas acuñaciones. Este culto se convirtió, como señaló De Siena, en el catalizador de la «rifondazione "ideologica" della polis»" ${ }^{39}$, una refundación que estuvo desencadenada por la precocidad de la conciencia política de los metapontinos, y cuya emergencia, ya en las últimas décadas del siglo VI a.C., ha dejado una huella inconfundible que todavía se puede rastrear en las fotografías aéreas: un cambio radical en la distribución de la tierra que debió traducirse en un serio debilitamiento del poder de las grandes familias ${ }^{40}$. El caso de Metaponto nos sirve para ilustrar el importante papel que desempeñó la religión délfica en la construcción del discurso de legitimación de los nuevos actores sociales. Por un lado, de la tiranía, tal y como podemos observar en el caso de Gelón de Gela y Siracusa ${ }^{41}$, y por otro, de la democracia, cuyo ejemplo más emblemático es Atenas $^{42}$. Sabemos muy poco acerca de la constitución política de Metaponto, pero lo más coherente con la información que ha llegado hasta nosotros es un proceso político que debió pendular de la tiranía a la democracia ${ }^{43}$. La institución del temenos de Apolo y Aristeas fue un reflejo de este desplazamiento de la «centralità politica», que supuso un «riduzione del ruolo istituzionale della religione tradizionale ${ }^{44}$.

\section{El temenos de Apolo y Aristeas en el contexto del conjunto monumental del ekklesiasterion}

La interpretación de Noe de que la base representada en la serie de Apolo era una reminiscencia del altar que podemos ver en la serie de Heracles no sirve para dar cuenta de la aparición de una pieza como la que estamos examinando. Si se tratara de un altar, no debería tener un agujero en su cara superior, cuya posición hace improbable que tuviera una función distinta a la de servir de punto de anclaje a la rama de laurel que sostiene Apolo. Lo que nos sugiere la evolución de las series de acuñaciones es otra cosa distinta. Es obvio que existe una cierta continuidad entre las representaciones de Aqueloo, Heracles y Apolo ${ }^{45}$. La serie de Heracles sirve de enlace

38 El patrocinio de Heracles sobre la actividad gimnástica es también sobradamente conocido, como apreció Noe, que escribió a propósito de estas acuñaciones: «It would be logical to conclude that the Heracles type, one of which shows that hero pouring a libation $\left(\mathrm{n}^{\circ} .312\right)$ may also have been connected with games» $(1931,7-8)$. Las dos divinidades más apreciadas en los gimnasios antiguos eran Hermes y Heracles. Cfr: Miller 2004, 50. Hermes aparece también representado en varias acuñaciones metapontinas, cuyas primeras emisiones datan de mediados del siglo IV a.C. Cfr. Johnston 1989, nº $3, \mathrm{n}^{\circ} 5, \mathrm{n}^{\circ} 42, \mathrm{n}^{\mathrm{o}} 54, \mathrm{n}^{\mathrm{o}} 71, \mathrm{n}^{\mathrm{o}} 77, \mathrm{y} \mathrm{n}^{\mathrm{o}} 80$.

39 De Siena 2001, 167-168 y 2001, 34.

40 De Siena 2001, 34; 2002, 35-36.

41 Scott 2014, 123.

42 Bowden 2005.

43 Hansen y Nielsen 2004, 280.

44 De Siena 1998, 168.

45 Continuidad que fue observada por Jannot, aunque esto le indujo a reordenar la cronología de las series, lo que no parece corresponderse con las características técnicas de las acuñaciones. Cfr. Jannot 1974, 302. 
con las otras dos. Sostiene una pátera en su mano derecha, como Aqueloo, y aparece representado junto a un altar, que recuerda a la base donde estaba fijada la rama de Apolo. Creo que esta continuidad es un reflejo de que todas estas representaciones compartían un mismo motivo, y el mejor candidato para este motivo común era la conmemoración de los juegos que probablemente desde época arcaica se celebraban en el ekklesiasterion de Metaponto. Si nos detenemos en esta continuidad, la palabra

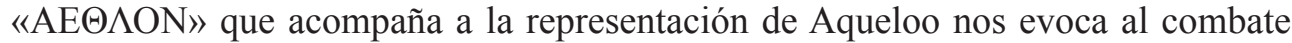
que el dios mantuvo con Heracles por la mano de Deyanira. Hasta ahora, muchos especialistas se han apoyado en la representación de Apolo contenida en las series de doble relieve para respaldar la tesis de la existencia real de una estatua de Apolo en el temenos de Apolo y Aristeas. Pero esta representación formaba parte de un grupo de acuñaciones en el que también estaban representados el dios Aqueloo y el héroe Heracles. Si había una escultura de Apolo, es probable que también las hubiera de los otros dos ${ }^{46}$. Estas esculturas podrían encontrarse dentro del ekklesiasterion, o distribuidas en la franja de ochenta metros que lo separaba del temenos de Apolo y Aristeas. La base que encontramos en la representación de la serie de Apolo, y que parece coincidir con la base representada en una de las series de Heracles, puede que formara parte de un grupo de piezas dispuesto originariamente junto a la imagen de cada divinidad, en honor de las cuales los ciudadanos, o tal vez, los atletas, las utilizarían para realizar libaciones ${ }^{47}$. Las palabras de Heródoto referidas a que la estatua con el nombre de Aristeas se alzaba al lado mismo de la imagen de Apolo se comprenden mejor si interpretamos que esta imagen formaba parte de una serie de esculturas que se espaciaban en la dirección del ekklesiasterion. La mención de Heródoto tendría, por tanto, un valor indicativo. Es decir, por un lado, habría dicho lo que Aristeas ordenó hacer a los metapontinos, que fue levantar una estatua en su propio nombre y un altar en honor de Apolo; y por otro, habría explicado dónde hicieron estas cosas, o sea, en el ágora, al lado de donde entonces (en tiempos de Heródoto) se encontraba la imagen de Apolo. Esto parece implicar que la imagen no formaba parte del temenos, pero es necesario recordar que en el momento de su visita a Metaponto el altar de Apolo y la escultura de Aristeas todavía no habían sido reunidos por un recinto exterior. No es que la imagen de Apolo no perteneciera a la estructura del temenos, sino que todos los elementos que enumera Heródoto pertenecían en realidad a la gran estructura del ekklesiasterion. Esta estructura contaba también con un segundo altar dedicado a Zeus, cuya relación con el ekklesiasterion ha sido más fácilmente identificada por su vecindad con su muro externo y por el hallazgo de dos cipos en los que constaba su advocación a Zeus Agoraios y Zeus Aglaios. La especial orientación en la que se encontraba el altar de Apolo (situado a solo ochenta metros en línea recta del corredor central del ekklesiasterion), y la posibilidad de que las esculturas de Apolo y Aristeas pudieran formar parte de un conjunto mayor, nos revelan que la estructura monumental del ekklesiasterion probablemente fuera más amplia de lo que habíamos pensado hasta ahora, y que constaba de dos altares para

46 Mertens-Horn ha llamado la atención sobre el notable parecido que existe entre la cabeza de una escultura de Apolo-Helios hallada en Metaponto y la acuñación de un estatero de plata que junto a la tradicional espiga de cebada, incluye una representación de la cabeza de la misma divinidad. Ambas piezas están datas en la segunda mitad del siglo IV a.C., y constituyen otro testimonio a favor de la tesis de que en ocasiones los metapontinos utilizaban esculturas reales como modelos de las divinidades que representaban en sus acuñaciones. Cfr. Mertens-Horn 2001, 83-85. Una segunda acuñación de Helios, en Johnston 1989, nº 53.

47 No se trataría, por tanto, de un altar destinado a sacrificios. 
sacrificios, y varias imágenes sagradas, frente a las que los atletas, o los ciudadanos, podían realizar libaciones en honor de los dioses. La enorme influencia de Delfos terminaría favoreciendo que una parte de este complejo adquiriese un uso propio relacionado con la mántica oracular. Este proceso debía estar ya en marcha en el momento de la visita de Heródoto (más o menos contemporánea a la serie de acuñaciones de doble relieve), y muy avanzado a mediados del siglo IV a.C., cuando oímos por primera vez hablar de «adivinos» a Teopompo. La consagración definitiva del temenos llegaría a finales del siglo III a.C., cuando se levantó el recinto exterior que delimitó el espacio sagrado del manteion.

Las series de acuñaciones de Apolo nos revelan también otro detalle importante en relación a la base que estamos analizando. Como hemos tenido ocasión de comentar, solo en una de ellas la rama de laurel aparece fijada a lo que acabo de describir como un altar de libaciones. La explicación de esta diferencia, y de las características de la pieza que nos ha llegado, tiene que estar relacionada con el hecho de que este pequeño altar de libaciones se encontrara en las inmediaciones de otro altar que también estaba dedicado a Apolo, pero cuya importancia no dejaba de crecer. Los metapontinos debieron trasladar el ritual de las libaciones de un altar a otro. Y no solo porque tuvieran una mayor preferencia por lo suntuoso, sino por una razón bastante más prosaica, con la que por desgracia están bastante familiarizados los historiadores del arte: los brazos de las estatuas de mármol tienden a romperse. Y si además, les hacemos sujetar una larga rama de laurel, sobrecargada con hojas de bronce, es solo una cuestión de tiempo que termine por imponerse la triste realidad de que ni siquiera el dios Apolo está por encima de las leyes de la física. Una vez apreciado el riesgo, y aprovechando la vecindad del segundo altar, lo más razonable era cambiar las libaciones de sitio, acortar la rama, y buscar una base donde fijar el tallo, para darle estabilidad y evitar la sobrecarga del brazo de la estatua. El elemento más apropiado para desempeñar este papel era el antiguo altar de libaciones, ahora sin uso, y sobre el que solo era necesario picar un agujero. De esta manera, el grupo de acuñaciones de Apolo se revela bajo una luz completamente inesperada. La secuenciación, tal y como la catalogó Noe, se muestra casi como una secuencia fotográfica de los cambios que se estaban produciendo en el temenos de Apolo y Aristeas. Los grabadores se convirtieron involuntariamente en corresponsales gráficos de la crónica de Heródoto.

¿Es posible que en lugar de varios altares de libaciones solo hubiera uno que se utilizara en honor de todos los dioses que formaban parte del grupo estatuario del ekklesiasterion? No sería extraño, dado que el grupo tenía un motivo común. El altar podría estar situado al principio, o al final, del recorrido que conectaba el ekklesiasterion con el temenos de Apolo y Aristeas, y las libaciones se realizarían en honor de Aqueloo, Heracles y Apolo. La única razón que induce a pensar que nos encontramos ante varios altares es que hubiera sido más difícil cambiar el uso del altar de Apolo si esto hubiera significado desproveer a las otras divinidades. Pero hay otra posibilidad que jugaría en favor de esta alternativa. Anteriormente he mencionado la existencia, a medio camino entre el ekklesiasterion y el temenos de Apolo y Aristeas, de un segundo recinto con dos bases en su interior. Una de estas bases podría haber sido la de un altar. El progreso en las excavaciones permitió a De Siena descartar que este segundo recinto fuera el temenos de Apolo y Aristeas, pero en su discusión con Adamesteanu sobre este asunto escribió unas palabras que merece la pena traer a colación aquí: «La doppia base si trova sulla grande plateia $\mathrm{N}-\mathrm{S}$ che separa i due 
spazi pubblici ed avrà ospitato qualche altro importante grupo statuario di cui non si hanno notizie ${ }^{48}$. ¿Es posible que el grupo al que se refiere De Siena estuviera constituido por las imágenes de Aqueloo y Heracles, reunidas finalmente en un sacelo, después de que la imagen de Apolo pasara a formar parte del manteion? Esta medida habría provisto a las esculturas de un nuevo altar, y se correspondería con la nueva asignación de la escultura de Apolo al altar de sacrificios que llevaba su nombre. Pero hay otras propuestas igualmente sugerentes para este sacelo. Mertens-Horn planteó que podría haberse construido para albergar una gran escultura de ApoloHelios conduciendo una cuadriga de caballos, lo que estaría apoyado por el hallazgo de la cabeza de esta escultura y el registro numismático, y además, se ajustaría mejor a la propuesta de datación del sacelo que realizó Mertens ${ }^{49}$. Pero ambas posibilidades son especulativas. Si el conjunto estatuario vinculado al ekklesiasterion estaba provisto de uno o varios altares de libaciones es algo sobre lo que solo podrá arrojar luz la aparición de un nuevo altar, de características similares al que fue representado en las series de acuñaciones.

\section{Conclusiones}

La primera conclusión de nuestra investigación es que debe considerarse seriamente la posibilidad de que la pieza hallada en el Parco Archeologico dell'area urbana di Metaponto sea efectivamente la base que aparece representada en la acuñación de Apolo, y por tanto, que nos encontramos ante una pieza de primer orden para nuestro conocimiento del temenos de Apolo y Aristeas. Esta conclusión tendría implicaciones importantes. Por un lado, aportaría una prueba decisiva de la existencia de la imagen de Apolo. Por otro, contribuiría a reforzar la tesis de que el resto de las imágenes representadas en el mismo grupo de acuñaciones se inspiró en un conjunto estatuario que se encontraba realmente en el ágora de Metaponto. En este aspecto, y más allá de la pieza concreta, el análisis ha revelado la fecundidad de aplicar a nuestra interpretación de las fuentes un método comparativo que tenga en cuenta las relaciones entre configuraciones de dioses y la red social y cultural que le otorgaba sentido $^{50}$. Es muy probable que toda el área comprendida entre el ekklesiasterion y el altar de Apolo formara parte de un mismo conjunto monumental. La asociación que Mertens y otros autores han establecido entre la estructura del ekklesiasterion y la celebración de certámenes corales y gimnásticos se compadece con la idea de que el grupo de acuñaciones que hemos examinado tuviera el mismo motivo. Pero es necesario añadir que esta clase de actividades estaba investida de un fuerte valor político y religioso, y que con independencia de cuál fuera su origen, experimentaron una enorme sacudida, y un gran impulso, como consecuencia de las transformaciones que a finales del siglo VI y principios del siglo V a.C. se produjeron en torno al Santuario de Delfos. La aparición del temenos de Apolo y Aristeas fue probablemente el resultado más notable de este impacto. Heródoto, con su gran olfato para las cosas importantes, aprovechó su visita a Metaponto para levantar acta de este acontecimiento tan extraordinario.

\footnotetext{
48 De Siena 1998, 159, n.45.

49 Mertens-Horn 2001, 85.

50 En la línea apuntada por Detienne 1998, 12-16.
} 


\section{Bibliografía}

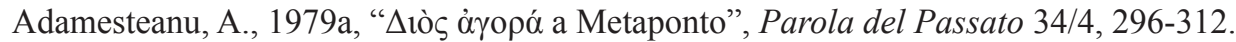

- 1979b, "Metaponto", en Gli Eubei in Occidente: Atti del diciottesimo convegno di studi sulla Magna Grecia, Taranto, 8-12 Ottobre 1978, Volumen 18, 350-352, Taranto.

Bolton, J. D. P., 1962, Aristeas of Proconnesus, Oxford.

Bowden, H., 2005, Classical Athens and the Delphic Oracle, Cambridge.

Bremmer, J., 1983, The Early Greek Concept of the Soul, Princeton.

Cerchiai, L., 2002, "Metaponto", en L. Cerchiai, L. Jannelli y F. Longo, Città greche della Magna Grecia e della Sicilia, 130-143, Venezia.

Colli, G., 1977, La Sapienza greca, vol. 1, Milano.

De Juliis, E., 2001, Metaponto, Bari.

De Siena, A., 1979, "Scavo del teatro-ekklesiasterion di Metaponto", en Gli Eubei in Occidente: Atti del diciottesimo convegno di studi sulla Magna Grecia, Taranto, 8-12 Ottobre 1978, Volumen 18, 357-366, Taranto .

- 1992, "Metaponto e il Metapontino", en L. de Lachenal (ed.), Da Leukania a Lucania. La Lucania centro orientale fra Pirro e $i$ Giulio-Claudii, 114-123. Roma.

- 1998, "Metaponto: problemi urbanistici e scoperte recenti", en E. Greco (ed.), Siritide e Metapontino. Storie di due territori coloniali, Atti dell'incontro di studio (Policoro 1991), 141-170, Naples-Paestum.

— 2001, "Profilo storico archeologico", en A. De Siena (ed.), Metaponto. Archeologia di una colonia greca, 7-44, Taranto.

- 2002, "Appunti di topografia metapontina", en G. Bertelli, D. Roubis (eds.), Torre di Mare I, Ricerche archeologiche nell'insediamento medievale di Metaponto (1995-1999), 25-40, Bari.

Detienne, M., 1998, Apollon le couteau à la main: une approche expérimentale du polythéisme grec, Paris.

Dodds, E. R., 1951, The Greeks and the Irrational, Berkeley and Los Angeles.

Fontenrose, J., 1980, Python: A Study of Delphic Myth and Its Origins, Berkeley.

Franke, P. R. y Hirmer, M., 1966, La Monnaie grecque, Paris.

Giacometti, D., 2005, Metaponto. Gli dei e gli eroi nella storia di una polis di Magna Grecia, Consenza.

Giannelli, G., 1963, Culti e miti della Magna Grecia. Contributo alla storia più antica delle colonie greche in occidente, Firenze.

Giardino, L. y De Siena, A., 1999, "Metaponto", en E. Greco (ed.), La città greca antica: istituzioni, società e forme urbane, 329-363. Roma.

Greco, E., 2006, “Agora e Zeus Agoraios”, en D. Morandi et al. (eds.), Tra Oriente e Occidente. Studi in onore di Elena Di Filippo Balestrazzi, 327-335, Padova.

Hansen, M. H. y Nielsen, T. H. (eds.), 2004, An Inventory of Archaic and Classical Poleis, Oxford.

Head, B. V., 1911, A Manual of Greek Numismatics, Oxford.

Jannot, J. R., 1974, "Intervento nel Dibattito", en Metaponto: Atti del tredicesimo Convegno di Studi sulla Magna Grecia, Taranto, 14-19 ottobre 1973, 300-303, Napoli.

Johnston, A., 1989, "The Bronze Coinage of Metapontum”, en Kraay-Mørkholm Essays. Numismatic Studies in memory of C. M. Kraay and O. Mørkholm, 121-136, Louvain-LaNeuve.

Knoepfler, D., 1988, “Sur les traces de l'Artémision d'Amarynthos près d'Érétrie”, Comptes rendus des séances de l'Académie des Inscriptions et Belles-Lettres, $1322^{\mathrm{e}}$ année, 2, 382-421. 
Lacroix, L., 1965, Monnaies et colonisation dans l'Occident grec, Bruxelles.

Lane, C. S., 2009, Archegetes oikistes, and new-oikistes: the cults of founders in Greek southern Italy and Sicily, Doctoral Thesis, Vancouver.

Lehmann, P. W., 1946, Statues on coins of southern Italy and Sicily in the Classical period, New York.

Mertens, D., 1979, "Il teatro di Metaponto", en Gli Eubei in Occidente: Atti del diciottesimo convegno di studi sulla Magna Grecia, Taranto, 8-12 Ottobre 1978, Volumen 18, 352356, Taranto.

- 1982, "Das Theater-Ekklesiasterion auf der Agora von Metapont", Architectura, 12, 93124.

- 1998, “L'archittettura e l'urbanistica di Metaponto nel quadro dell'economia locale e dell'evoluzione générale nella Magna Grecia", en E. Greco (ed.), Siritide e Metapontino. Storie di due territori coloniali, Atti dell'incontro di studio (Policoro 1991), 123-140, Naples-Paestum.

- 2001, "L'Architettura", en A. De Siena (ed.), Metaponto. Archeologia di una colonia greca, 745-70. Taranto.

- 2006, Città e monumenti dei greci d'occidente: dalla colonizzazione alla crisi di fine V secolo a.C, Roma.

- y Greco, E., 1996, "Urbanistica della Magna Grecia”, en G. Pugliese Carratelli (ed.), I Greci in Occidente, 243-262, Milano.

Mertens-Horn, M., 2001, “La scultura di marmo", en A. De Siena (ed.), Metaponto. Archeologia di una colonia greca, 71-88. Taranto.

Miller, S. G., 2004, Ancient Greek Athletics, New Haven.

Noe, S. P., 1931, The Coinage of Metapontum. Part 2. New York.

Poole, R. S., 1873, A Catalogue of The Greek Coins in The British Museum. Italy, London.

Rutter, N. K., 2001, Historia numorum. Italy, London.

Scott, M., 2014, Delphi: A History of the Center of the Ancient World, Princeton, New Jersey.

Stazio, A., 1974, "Osservazioni sulla monetazione di Metaponto", en Metaponto: Atti del tredicesimo Convegno di Studi sulla Magna Grecia, Taranto, 14-19 ottobre 1973, 67-106. Napoli.

- 1999, "Le emissioni monetarie dei centri Greci", en D. Adamesteanu (ed.), Storia della Basillicata. 1. L'Antichità, 455-469, Roma.

Uggeri, G., 1969, "K $\lambda \tilde{\eta} \rho 0$ arcaici e bonifica classica nella $\chi \tilde{\omega} \rho \alpha$ di Metaponto", Parola del Passato, 24/1, 51-71. 